\title{
A new and simple variable-angle accessory for infrared specular reflectance
}

\author{
M. C. Polo ${ }^{\text {a) }}$ \\ Departament de Física Aplicada i Òptica, Universitat de Barcelona, Av. Diagonal 647, \\ E-08028 Barcelona, Spain \\ N. Ferrer, M. Romero, J. Pérez, M. Quevedo and F. Vilardell \\ Serveis Cientificotècnics, Universitat de Barcelona, Lluís Solé i Sabarís 1, E-08028 Barcelona, Spain
}

(Received 2 July 1998; accepted 18 September 1998)

\begin{abstract}
A simple, low-cost accessory (patent pending) with only two flat mirrors and a new variable-angle mechanism has been developed for infrared specular reflectance measurements. The system allows the angles of incidence to be varied continuously from $15^{\circ}$ (near normal incidence) to $85^{\circ}$ (near grazing angle) without losing the alignment of the accessory. The reflectivity of boron nitride thin films deposited on metallic substrates has been measured at different angles of incidence to demonstrate the utility of this accessory. (C) 1999 American Vacuum Society.
\end{abstract}

[S0734-2101(99)02001-1]

\section{INTRODUCTION}

Infrared specular reflection spectroscopy (IRSR) is a simple, nondestructive technique for the chemical and physical analysis of surfaces and thin films. It provides, for example, the refractive index of the film, which can be related to its composition ${ }^{1}$ and is also useful to observe the vibrations of surface adsorbed species. ${ }^{2}$ Moreover, when the samples are not infrared transparent, such as coatings on metallic substrates, infrared measurements must be done in reflection mode. IRSR spectroscopy is also used to examine the structure of anisotropic thin films ${ }^{3}$ because, besides the transverse optic component of the vibrational modes (TO), the longitudinal optic component (LO) can also be observed. These features can be greatly enhanced when performing experiments under wide incident angle conditions. ${ }^{4}$

Several accessories for specular reflectance analysis are available commercially. There are two kinds of accessories: those in which samples are analyzed at a fixed angle of incidence (with prices ranging between $\$ 500$ and $\$ 1000$ ) and variable angle specular reflectance accessories (VASR). The latter allow the angle of incidence to be changed typically within a range of $30^{\circ}-80^{\circ}$, and the involve many optical elements (up to eight mirrors, flat and paraboloid). This put up the price of commercial VASR accessories which could cost up to $\$ 5000$. Another drawback is that the mirrors need to be realigned when the angle of incidence is changed.

In this note, we describe a versatile accessory for most reflectance measurements since the angle can be varied from near normal incidence $\left(15^{\circ}\right)$ for highly reflective surfaces to near grazing angle $\left(85^{\circ}\right)$ for extremely thin films. It consists of only two mirrors that are moved simultaneously with the sample by means of a novel variable-angle mechanism. The present design makes this accessory a very reasonable alternative to commercially available ones.

Finally, we illustrate the use of this accessory in measuring the reflectivity spectra of boron nitride coatings on me-

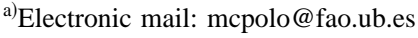

tallic substrates at different indidence angles in order to study the structure of the films.

\section{DESIGN AND PERFORMANCE CHARACTERISTICS}

The accessory consists of a wedge that is moved along the $y$ axis by a screw rod. Its position at angles of incidence of $15^{\circ}, 45^{\circ}$, and $85^{\circ}$ are shown in Fig. 1 . The sample holder is connected to the wedge and therefore they move together. Two flat mirrors are located perpendicularly to the surface of both (part 4), which act as mirror holders. These holders can rotate around the pivot screws (3), which are the only fixed points of the accessory. When the wedge moves down (Fig. 2), the two roller bearings (part 5) slide along the contour of the wedge and the relative height between the center of the mirror and the sample $(h)$ decreases, while the distance between mirrors $(2 d)$ increases. Therefore the angle of incidence $\alpha$ on the sample varies according to $d=\operatorname{tg} \alpha-h$. In such a system where $d$ and $h$ change simultaneously, the beam remains focused onto the surface of the sample when the angle of incidence changes. Figure 2 illustrates the design of the wedge contour, which is a function of $h$ and $d$. The tangent point between the roller bearing and the wedge determines the incident angle on the sample. The screw rod has a graduated scale corresponding to the angle of incident light on the sample.

When using grazing angles, the diameter of the beam must be reduced because its spot on the mirror enlarges. An iris located before the first mirror allows the beam to fit on it. On the other hand, a set of black masks allow to position very small samples on the accessory. Samples are placed horizontally over the sample holder, which is convenient in case of fragile samples. The configuration of the accessory has the possibility to adapt a polarizer before the incidence of the beam on the first mirror.

The above description points out the simplicity of the accessory. In addition, the small size of the accessory and the ease of manipulation, makes it possible to couple it to any 


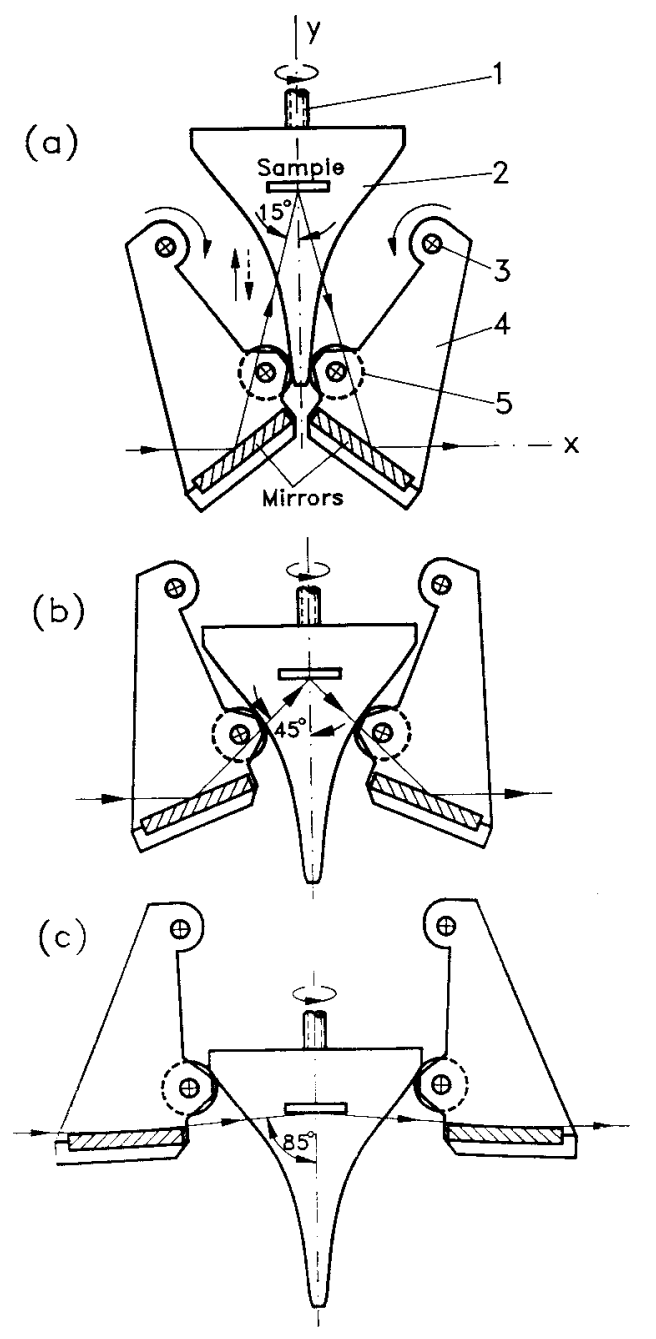

FIG. 1. Side view of the new variable angle accessory at three different positions corresponding to three incidence angles: (a) $15^{\circ}$, (b) $45^{\circ}$, and (c) $85^{\circ}$. ( 1 screw rod, 2 wedge, 3 pivot screw, 4 mirror holder, 5 roller bearing.)

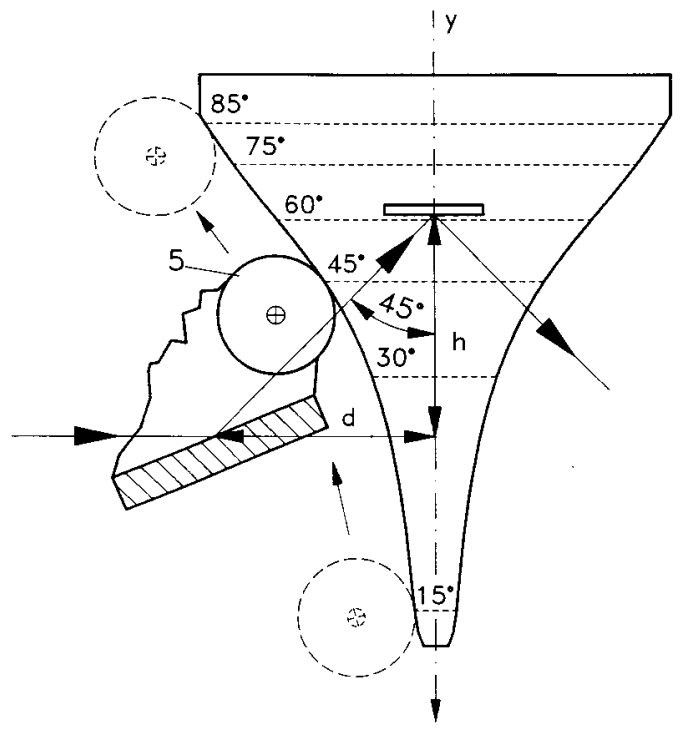

FIG. 2. Schematic drawing of the contour wedge and the roller bearing showing its relative position depending on the incident angle.

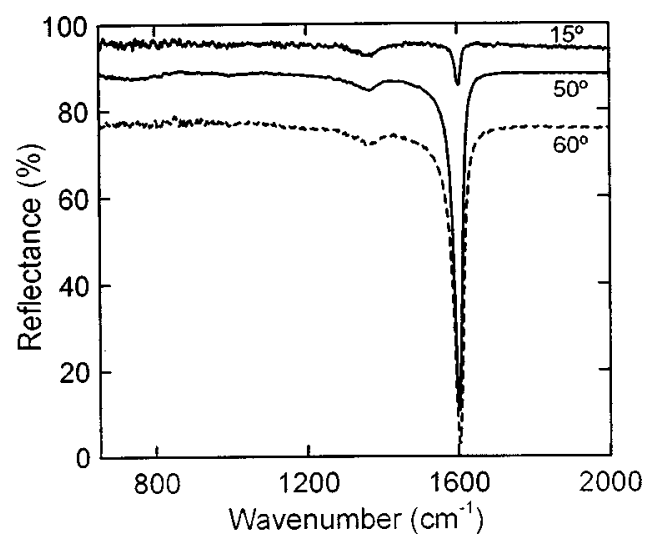

FIG. 3. Experimental reflectance spectra at different incidence angles for a 130-nm-thick hexagonal BN thin film deposited on a $\mathrm{NiCr}$ coated silicon.

kind of spectrometer. Moreover in equipment that operates under vaccum, it is possible to mount the screw rod, and therefore the scale, outside the sample compartment, in such a way that it is not necessary to break the vacuum to change incidence angle.

\section{APPLICATION TO THE OPTICAL STUDY OF BN FILMS}

We have applied our homemade accessory to the IRSR analysis of hexagonal boron nitride ( $h$ - BN) thin films. It was performed using a DA3 BOMEM Fourier transform infrared (FTIR) spectrometer in the wave number range 500-4000 $\mathrm{cm}^{-1}$ with a resolution of $4 \mathrm{~cm}^{-1}$. The films were prepared by radio frequency plasma-enhanced chemical vapor deposition on NiCr-coated silicon substrates kept at $300{ }^{\circ} \mathrm{C} .{ }^{5}$ As the optical properties of $h$-BN are anisotropic, different vibration modes can be excited depending on both, the orientation of the $s p^{2}$-bonded basal planes with respect to the surface substrate and the polarization of the light. ${ }^{3}$ The reflectivity spectra of a $130 \mathrm{~nm} \mathrm{BN} \mathrm{film,} \mathrm{for} p$ polarization, performed at different incidence angles are shown in Fig. 3. The spectrum taken at near normal incidence $\left(15^{\circ}\right)$ shows the TO and LO components of the in-plane stretching vibration $E_{1 u}\left(\omega_{\mathrm{TO}}\right.$ $=1380 \mathrm{~cm}^{-1}, \omega_{\mathrm{LO}}=1600 \mathrm{~cm}^{-1}$ ) but there is no signal from the out-of-plane bending mode $A_{2 u}\left(\omega_{\mathrm{TC}}=780 \mathrm{~cm}^{-1}, \omega_{\mathrm{LO}}\right.$ $\left.=828 \mathrm{~cm}^{-1}\right)$. The LO coupling with the incident electric field is strongly enhanced when the angle of incidence increases. At an incidence angle of $50^{\circ}$, the sharp feature at $1600 \mathrm{~cm}^{-1}$ and the absence of the LO component of the low energy mode indicate that the films have the $c$-axis contained in the plane parallel to the film surface. ${ }^{3}$ However, when one uses wider angles of incidence (above $50^{\circ}$ ) the reflectivity is reduced, so the sensitivity to detect LO modes decreases.

\section{CONCLUSION}

A new variable-angle specular reflectance accessory based on a novel mechanism has been fabricated. The simultaneous movement of the mirrors and the sample allows the incidence angle to vary from $15^{\circ}$ to $85^{\circ}$, avoiding the need for realignment when changing the angle. The accessory has been succesfully applied in the measurement of the infrared 
reflectivity of boron nitride thin films. The ease and low cost of the manufacture of this accessory make it a valuable alternative to commercial ones.

\section{ACKNOWLEDGMENT}

The authors are grateful to Dr. J. L. Andújar for supplying the boron nitride thin films.
${ }^{1}$ M. Ben el Mekki, N. Mestres, J. Pascual, E. Pascual, M. C. Polo, and J. L. Andújar, Diamond Relat. Mater. 7, 365 (1998).

${ }^{2}$ Y. Kobayashi and T. Ogino, Surf. Sci. 368, 102 (1996).

${ }^{3}$ M. C. Polo, M. Ben el Mekki, J. L. Andújar, N. Mestres, and J. Pascual, Diamond Relat. Mater. 6, 1550 (1997).

${ }^{4}$ A. Goullet, J. Camassel, L. Martín, J. Pascual, and E. Philippot, Phys. Rev. B 40, 5750 (1989).

${ }^{5}$ J. L. Andújar, E. Bertran, and M. C. Polo, J. Vac. Sci. Technol. A 16, 578 (1998). 